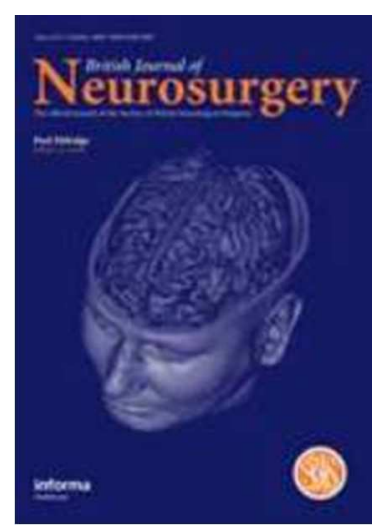

\title{
Long-term pain relief at five years after medical, repeat surgical procedures or no management for recurrence of trigeminal neuralgia after microvascular decompression: analysis of a historical cohort
}

\begin{tabular}{|r|l|}
\hline Journal: & British Journal of Neurosurgery \\
\hline Manuscript ID & CBJN-2018-0383 \\
\hline Manuscript Type: & Original Article \\
\hline Date Submitted by the Author: & $28-J u l-2018$ \\
\hline Complete List of Authors: & $\begin{array}{l}\text { Jafree, Daniyal; University College London, MB PhD Programme, Faculty of } \\
\text { Zakrzewska, Joanna; UCLH NHS Foundation Trust, Eastman Dental } \\
\text { Institute }\end{array}$ \\
\hline Keywords: & Microvascular decompression, Trigeminal neuralgia, Recurrence, Treatment \\
\hline &
\end{tabular}

\section{SCHOLARONE ${ }^{\text {tw }}$}

Manuscripts 


\title{
Long-term pain relief at five years after medical, repeat surgical procedures or no management for recurrence of trigeminal neuralgia after microvascular decompression: analysis of a historical cohort
}

\author{
Daniyal J Jafree ${ }^{1 *}$, Joanna M Zakrzewska ${ }^{2}$ \\ ${ }^{1}$ MB PhD Programme, Faculty of Medical Sciences, University College London, London, UK \\ ${ }^{2}$ Oral Medicine Unit, Eastman Dental Institute, UCLH NHS Foundation Trust, London, UK \\ * Corresponding author \\ Daniyal J Jafree \\ MB PhD Programme, \\ Faculty of Medical Sciences, \\ University College London, \\ Gower Street, \\ London, \\ WC1E 6BT, United Kingdom \\ Telephone: $+44(0) 7748417402$ \\ Email: daniyal.jafree.13@ucl.ac.uk
}

Keywords: Microvascular decompression; Trigeminal neuralgia; Recurrence 


\section{ABSTRACT}

Background: Management strategies for the recurrence of trigeminal neuralgia after microvascular decompression include repeat procedures, medical management or no further therapy. No consensus exists as to which strategy is best for pain relief. The aim of this study was to determine the characteristics of patients with recurrences after microvascular decompression in the cohort, and to compare long-term pain relief between different management strategies.

Materials and methods: A historical cohort of patients who underwent microvascular decompression at a neurosurgical institution between 1982-2002, followed up by postal survey at five years, was included. Characteristics of patients who experienced a recurrence were compared to those who were recurrence free, and pain relief was compared between each management strategy.

Results: From 169 responders who were included in the study, 28 (16.6\%) experienced a recurrence after MVD. No characteristics were significantly different between patients who experienced a recurrence and those who did not. Repeat procedures, including repeat microvascular decompression, partial sensory rhizotomy or radiofrequency thermocoagulation, yielded the highest proportion of pain relief after recurrence $(p=0.031)$, with $63.6 \%$ of patients pain-free at five-years. There was no evidence to suggest that the choice of repeat procedure influenced the likelihood of pain relief after recurrence. No further treatment yielded 57.1\% painfree, whereas medical therapy had the lowest proportion of pain free patients, at $10.0 \%$.

Conclusion: A variety of options are available to patients for recurrence of $\mathrm{TN}$ after microvascular decompression with repeat procedures yielding the greatest likelihood of long-term pain relief in this historical cohort. The choice of management should consider the mechanism of recurrence, the benefits and risks of each option and the severity of the pain. Regardless of the management strategy selected, careful phenotyping of patients before and after surgery is paramount. 


\title{
INTRODUCTION
}

Microvascular decompression (MVD) is the most common neurosurgical approach for classical trigeminal neuralgia (TN) with neurovascular compression (NVC). MVD has a risk of mortality below $0.3 \%$, and above $80 \%$ of patients remain pain-free without need for further treatment. ${ }^{1,2}$ Within the first four post-operative years, MVD provides the best results of all procedures, whilst reducing the risk of complications that come with ablative procedures. ${ }^{3}$ These, in addition to its safety in elderly patients, ${ }^{4}$ make MVD an attractive option for the definitive treatment of TN.

A proportion of patients with TN experience recurrence of their pain after the procedure. Approximately $4 \%$ of patients per year experience recurrence of $\mathrm{TN}$ after MVD, dropping to 1-2\% per year by the sixth year after surgery. ${ }^{5}$ Over a period of 10-20 years, the recurrence rate of TN after MVD may exceed $10 \%{ }^{3}$ Recurrence after MVD is caused by new or additional NVC, formation of arachnoid adhesions or granuloma and slippage of padding. ${ }^{6-9}$ Though the proportion of patients experiencing recurrence is considerable, there is no consensus as to its optimal management. Repeat procedures may include invasive options such as repeat MVD and partial sensory rhizotomy (PSR), or percutaneous options such as radiofrequency thermocoagulation (RFT and glycerol injection (GI) which are less invasive. Some patients opt out of further treatment, or choose medical therapy alone. A few small cohort studies compare outcomes between the different management strategies after a recurrence, ${ }^{9-13}$ but a comparison of different surgical interventions, including invasive or percutaneous procedures, against medical therapy and no further treatment is required. This complicates the decision process for patients and clinicians when deciding between different treatments. ${ }^{14}$

Our team has access to a historical database, prospectively maintained and gathered from one neurosurgeon's entire posterior fossa surgical practice between 1982 and 2002. This database has been previously reported in terms of patient satisfaction and post-operative quality of life. ${ }^{15,16}$ A systematic assessment of recurrences after MVD in this cohort would both add to the paucity of literature comparing treatment options after recurrence, and inform future reports as to whether repeat procedures, medical management or no therapy at all is best.

\begin{abstract}
Aims and hypothesis
The aim of this study was to define the characteristics and determine management and pain outcome of patients with a recurrence after first MVD in our cohort. We hypothesize that patients who experience a recurrence after MVD will have distinguishable characteristics pre-operatively, and that repeat operations, including invasive or percutaneous procedures, will provide better pain relief than medical management or no therapy.
\end{abstract}




\section{METHODS}

\section{Participants and eligibility}

Ethical approval was given by Frenchay Hospital Bristol LREC (Project Number: 2001/60). Patients were drawn from one neurosurgeon's entire posterior fossa surgery practice from $1982-2002$, reported in terms of satisfaction, quality of life and used to validate and design a patient-experience assessment set. ${ }^{15,17}$ All patients who underwent MVD for primary idiopathic TN, diagnosed using the second iteration of the International Classification for Headache Disorders, were included. ${ }^{18}$ Patients were excluded: with a secondary cause of trigeminal neuralgia including malignancy or multiple sclerosis, with a concurrent cranial nerve disorder, or with a failure of their MVD. Failure was defined as incomplete pain relief after MVD, requiring medication to obtain pain relief or surgery within 3 months after initial operation. Patients who had undergone prior procedures for $\mathrm{TN}$ were separated from the cohort and analysed independently.

\section{Data collection}

Patients were followed up by postal survey at a mean of five years postoperatively (with a standard deviation of 3.8), using a comprehensive assessment set for patient experience and postoperative complications. ${ }^{17}$ Prior to that they had annually completed a short one page questionnaire returned to the department. Responses were returned to the independent observers including one physician and one neurosurgeon. Recurrence of TN was defined as any post-operative pain at least three months after MVD. Recurrence was classified as major (severe pain requiring drugs or repeat procedures) or minor (transient, mild pain, resolving within 1-2 months). Characteristics extracted from healthcare records include: previous procedures, age at operation and onset of $\mathrm{TN}$, gender, duration of symptoms, division of trigeminal nerve involved, side of pain, operative findings and whether compression was arterial, venous or mixed, postoperative pain relief, presence of and time to recurrence and second treatment. The management strategy for recurrence was also collected from the assessment set, including repeat procedures (invasive procedures such as MVD or PSR and percutaneous procedures such as RFT or GI), medical management (patients taking drugs licensed for TN) or no management (opting for natural remission with no further procedures or medication). The outcomes, as determine by the assessment set, was whether the patient was pain free or not, taking drugs for control of TN and the frequency of drug use at the time of follow-up. Data was anonymised and entered on a spreadsheet.

\section{Data analysis}

Comparison of the characteristics between patients with a recurrence and those without were performed using Mann-Whitney $U$ and $\chi^{2}$ tests. Contingency analyses to compare outcomes between management strategies after recurrence were performed using a $\chi^{2}$ test, or Fisher's exact test where cells had a value less than 5 . All data were managed and statistics performed using IBM SPSS Statistics for Macintosh Version 24.0 (IBM 
1

2

3

4

5

6

7

8

9

10

11

12

13

14

15

16

17

18

19

20

21

22

23

24

25

26

27

28

29

30

31

32

33

34

35

36

37

38

39

40

41

42

43

44

45

46

47

48

49

50

51

52

53

54

55

56

57

58

59

60

Corp., Armond, NY). All continuous variables were found to have a skewed distribution, and median values are reported with interquartile ranges (IQR). $p$ values less than or equal to 0.05 were considered statistically significant. Graphs were drawn using Prism for Macintosh, Version 7 (GraphPad Software Inc., San Diego, CA). 


\section{RESULTS}

\section{Study participants and characteristics}

From 1982 to 2002, 284 patients underwent MVD for TN at the neurosurgical centre and were sent assessment sets at a mean follow-up of five-years (Fig. 1). From these, 64 (22.5\%) were excluded due to: death, non-response, lack of postal address or telephone-follow up only. From the remaining 220 patients who responded to the survey, $18(8.2 \%)$ were excluded due to failure of MVD. $33(15.0 \%)$ responders had previous procedures for relief of TN, and were separated from the cohort and analysed independently. For the main analysis, this left 169 (59.5\%) patients for inclusion from the original MVD cohort.

Twenty eight (16.6\%) patients experienced a recurrence of their TN within the five-year period after MVD, including 18 major recurrences and 10 minor recurrences. The characteristics of patients with a recurrence after MVD were compared with those who were recurrence free (Table 1). Some variables had missing data. Categorical data with missing variables are denoted with denominators. Continuous variables with missing data included age of onset of symptoms (recurrence, $\mathrm{n}=4$; recurrence-free, $n=$ 34) and duration of symptoms (recurrence, $n=4$; recurrence-free, $n=36$ ). No characteristics were significantly different between patients with a recurrence after MVD and those recurrence-free. The median time to recurrence was 15.7 months (IQR; 8.0-44.2), with $52.0 \%$ of these occurring within the first 18 months postoperatively. In all patients, arterial compression was the most common surgical finding, and this did not differ whether the patient experienced a recurrence or not. Details of the vascular findings at operation in patients are provided in Supplementary Table 1. The 64 non-responding patients had a significantly higher age at diagnosis $(U=6280.0 ; p=0.019)$ and consequently at surgery $(U=$ 8243.0; $p=0.032$ ) compared to 220 who responded.

\section{Management strategies and pain relief at five years for recurrence after MVD}

At a mean five year follow up, the choices that patients made for treatment of recurrence and their effect upon pain relief were compared. From 18 patients with a severe recurrence, four $(22.2 \%)$ opted for no further treatment, four $(22.2 \%)$ opted for medical management alone and 10 (55.6\%) opted for repeat procedures, including three MVDs, three PSRs and four RFTs. From the 10 patients with a minor recurrence, three $(30.0 \%)$ opted for no further treatment, six $(60.0 \%)$ opted for medical management and one $(10.0 \%)$ opted for an RFT. By the end of the five-year follow up period, 12 out of $28(42.9 \%)$ patients with a recurrence were pain-free. Seven $(25.0 \%)$ were using medication for control of pain, of 
which five (17.9\%) were using medication once daily and two (7.1\%) used medication twice or more per day. The severity of recurrence did influence the likelihood of being pain-free or requiring drugs to control pain at five years.

The difference in proportion of patients who were pain-free between no treatment, medical therapy and further procedures varied significantly $\left(\chi^{2}=6.9, p=0.031\right.$; Fig. 2). Repeat procedures yielded the highest proportion of pain-free patients $(7 / 11 ; 63.6 \%)$, including repeat MVD $(2 / 3 ; 66.7 \%)$, PSR $(2 / 3 ; 66.7 \%)$ or RFT $(3 / 5 ; 60.0 \%)$. After repeat procedures, no further treatment yielded the next highest proportion of pain-free patients $(4 / 7 ; 57.1 \%)$, whereas medical therapy had the lowest proportion $(1 / 10 ; 10.0 \%)$. The proportion of patients using medication to relieve pain at five-year follow up did not vary significantly between no treatment, medical therapy or repeat procedures $\left(\chi^{2}=2.8, p=0.25\right)$. The frequency of drug use also did not vary significantly between these groups $\left(\chi^{2}=5.8, p=0.21\right)$. The outcomes for patients with failure of MVD are provided in Supplementary Fig. 1).

\section{Outcomes in patients with prior procedures for $\mathbf{T N}$}

Of the 33 patients with procedures for TN prior to MVD, from which 16 were peripheral surgery (cryosurgery or neurectomy of peripheral branches) and 17 were performed at the Gasserion ganglion level, $11(33.3 \%)$ experienced a recurrence after the subsequent MVD, including 4 with peripheral surgery and 7 at the Gasserion ganglion level. This was a significantly higher proportion than the 28 out of 169 patients without prior procedures who experienced a recurrence after MVD $\left(\chi^{2}=5.0\right.$, odds ratio; $2.5,95 \% \mathrm{CI} ; 1.1-3.5, p=0.026)$. Eight of the 11 recurrences in patients with prior procedures were major, and three were minor. There were no significant differences in characteristics between the study cohort and those who had prior surgery. Only two out of the 11 patients who had prior surgery experienced a recurrence were pain-free at five-year follow-up (Supplementary Fig. 2). Patients with a recurrence after MVD who had undergone prior surgery required an increased frequency of drug use for pain relief $(\mathrm{OR}=6.0 ; 95 \% \mathrm{CI}, 1.2-29.7$; Fisher's exact test; $p=0.041)$, compared to those who had a recurrence after MVD as their first procedure. There were no significant differences in pain relief between the different treatment options for patients who experienced a recurrence with prior surgery. 


\section{DISCUSSION}

In this analysis of recurrences after MVD in our historical cohort, no clear characteristics distinguished patients who experience a recurrence of TN after MVD within five years. Patients who experience a recurrence opt for a variety of strategies including medical therapy, repeat procedures or no therapy at all. Repeat procedures, including MVD, PSR or RFT, yielded the best pain relief, followed by no further treatment, with medical management as the worst option for pain relief. There was no evidence to suggest that the choice of procedure influenced the likelihood pain relief. Patients with a prior procedure had poorer pain relief, and the various management strategies did little to change this. This may have been due to addition nerve injury from the procedure itself.

To our knowledge, this is the first study to compare different surgical options to medical management or no therapy for the treatment of recurrence after MVD. Though the database is historical, it was prospectively maintained, meaning that even those with failed MVD, who were excluded from final analyses, were followedup at five years. This study is not without limitations. The rarity of $\mathrm{TN}^{3}{ }^{3}$ in addition to the $16.6 \%$ of patients who experienced a recurrence in this study, resulted in a small sample size, which limits the interpretability of contingency analyses, prohibits the use of a regression model to determine predictors of recurrence and prevents a thorough comparison between invasive procedures, such as PSR or MVD, with percutaneous procedures such as PSR. The older criteria used for diagnosis in this study have been superseded by more contemporary ones, which differentiate TN into different subtypes. ${ }^{19,20}$ As different subtypes of TN, which may not have been picked up by the study, have different responses to surgery, ${ }^{21-24}$ this may confound analyses. The degree of vascular compression may be a major prognostic factor for pain outcomes. ${ }^{21}$ At the time of posterior fossa procedures in these patients, intraoperative detail such as the degree of distortion on the trigeminal nerve and its effect on the nerve's appearance were not appreciated, and so were not available for this study. Non-responders had a significantly greater age at diagnosis and surgery compared to the cohort, predominantly due to death or no answer to postal survey, meaning that outcomes of some of the older population may have been missed.

Female gender, duration of TN greater than 8 years, memorable onset, venous compression and lack of immediate pain relief have been associated with recurrence of TN after surgery. ${ }^{11,21-26}$ However, in agreement with one study, we found no such characteristics that defined patients with a recurrence. ${ }^{21}$ Compression can occur at multiple sites but the exact location of the compression may be an important factor in determining recurrence. It has been postulated that the anatomical location of transition between central and peripheral myelinated fibres, termed the root entry zone (REZ), may be at risk of compression by pulsatile vessels. Two 
prospective observational studies have demonstrated that patients with significant atrophy and compression of the REZ, as noted on MRI are likely to benefit the most from MVD. ${ }^{27,28}$ High quality MRI were not available at the time this study was done and so the precise location of the compression cannot be confirmed. This may account for the findings from this study that the predominant vessels causing NVC are arterial, but that higher recurrences were not found in those with venous compression, as previously suggested by others who, at the time, may not have had access to high quality MRIs. ${ }^{5,24}$ There appears to be consensus that a repeat MVD is a viable option for patients with a recurrence, albeit pain relief and numbness are significantly worse for repeat compared to primary MVD..$^{6,8,29,30}$ Other studies of repeat procedures after recurrence have shown good results for partial nerve sectioning, rhizotomy and stereotactic radiosurgery. ${ }^{9,10,13}$ These operations yield better pain relief after MVD than after other destructive procedures, ${ }^{13}$ so the type of prior procedures in patients is an important factor to consider. Tyler-Kabara and colleagues found that prior destructive procedures were a negative predictor for short-term, but not long-term pain relief postoperatively, ${ }^{23}$ with Rath and colleagues also showing poor outcomes for repeat destructive procedures. ${ }^{11}$

Medical management of recurrences was worse for pain relief when compared to repeat procedures. A case series following up 80 patients treated with MVD found, that in five patients with major recurrence of TN, none were amenable to medical management. ${ }^{12}$ The discontinuation of therapy was shown to yield satisfactory outcomes, and to our knowledge this is the first time that no therapy has been shown to be nearly as efficacious as repeat procedures for management of a recurrence. Some patients may opt for no medical therapy as the side effects of the medications may outweigh their benefit. Conversely some who have had a recurrence may be reluctant to stop all medication in case of a more severe recurrence.

The disparity in predictors for recurrence of TN after MVD may be due to different phenotypes of TN within a study population. Burchiel and colleagues have advocated dividing patients into type 1 and type 2, with the latter having a prolonged background pain for more than half of the time. ${ }^{31}$ It is hypothesised that patients with TN type 2 may have a higher recurrence rate. Reports using this classification show poorer outcomes for TN type 2 after MVD or stereotactic radiosurgery. ${ }^{22,32,33}$ However, Sindou and colleagues found no difference in outcomes after surgery between classical TN and TN with a baseline of permanent pain, which they defined as atypical TN. ${ }^{32}$ It is proposed that the mechanism of continuous pain observed in TN phenotypes in these studies may differ from the paroxysmal pain of $\mathrm{TN},{ }^{20}$ underlying the variable response to surgery and the heterogeneity of predictors for recurrence. It is thus essential to carefully phenotype all patients with TN, both clinically and using high quality imaging whose sensitivity and specificity is improving, ${ }^{35}$ and to use the most contemporary diagnostic criteria such that studies are comparable and data reproducible. ${ }^{19}$

When choosing treatment options after MVD, three factors should be taken into consideration. Firstly, the mechanism of return to pain may guide treatment. The failure of medical management indicates a mechanical 
fault that may not be amenable to contemporary pharmacological therapies. The mechanism of recurrence may be deduced by intraoperative findings and imaging, to guide choice of repeat procedure. For example, in the absence of NVC, Ishikawa and colleagues propose that arachnoid thickening can lead to distortion between the root and surrounding structures. ${ }^{36}$ Sindou and colleagues found that focal arachnoiditis results in poorer outcomes. These arachnoid changes could result movement and hyper-excitability of the nerve. As such, Revuelta-Gutierrez and colleagues suggest that all the arachnoid is freed, and mild neuropraxia induced by compressing bipolar tips at the root entry zone. ${ }^{37}$ However, when NVC is found, a repeat MVD may be more appropriate. The second factor to be taken into consideration when choosing treatment options is the additional complications that may arise. For example, the sensory loss associated with destructive procedures is common, ${ }^{36,37}$ and its impact on quality of life can be significant and should be communicated to patients. ${ }^{16,38,39}$ Thirdly and finally, the severity of recurrence should be determined by the patient, considering the option of no treatment at all, which may result in good pain relief in the long-term whilst also avoiding the complications and risks associated with neurosurgery. Whether repeat procedures, medical management or no treatment is chosen, the management for a recurrence after MVD should take into consideration risks and benefits of each option. These should be made clear to the patient to ensure informed consent and a shared-decision making process.

\section{Conclusion}

Recurrence after MVD is a common issue that is difficult to manage, though its predictors are unclear. There are a variety of options for the management of a recurrence, including repeat procedures, medical or no treatment. Our study indicates that repeat procedures, including repeat MVD, PSR or RFT yield the greatest likelihood of pain relief, and medical management the worst. Those who experience a recurrence after previous procedures had poor outcomes in this cohort. Ultimately, the decision-making process for a recurrence after MVD requires careful phenotyping before and after surgery, consideration of the mechanism of recurrence and balance of the benefits and risks of each treatment strategy.

Acknowledgements: The authors are grateful to Professor Hugh Coakham's (Frenchay Hospital, Bristol), whose entire prospective posterior fossa surgery practice from 1982 - 2002 this study represents. JZ was supported by the National Institute for Health Research University College London Hospitals Biomedical Research Centre. Jillie Abbott, a patient from Trigeminal Neuralgia UK, inputted all the data and assisted with arbitration. Ben Lopez and Sung Kim helped with the initial analysis of the data and were authors on previous publications. The authors are grateful to UCL AcaMedics for linking DJ to the project.

Declaration of interest: The authors have no known conflicts of interest to declare. Both authors contributed to the design of the study. JZ designed and distributed the assessment set and organised its data entry. DJ analysed the data and wrote the first draft. All authors contributed equally to the subsequent revision and 
acceptance of the final manuscript, and are accountable for all aspects of the study.

Funding: No funding was received for this research.

\section{REFERENCES}

1. Xia L, Zhong J, Zhu J et al. Effectiveness and Safety of Microvascular Decompression Surgery for Treatment of Trigeminal Neuralgia: A Systematic Review. J Craniofac Surg 2014;25:1413-1417

2. Wang DD, Ouyang D, Englot DJ et al. Trends in surgical treatment for trigeminal neuralgia in the United States of America from 1988 to 2008. J Clin Neurosci 2013;20:1538-1545

3. Zakrzewska JM, Linskey ME. Trigeminal neuralgia. BMJ 2014;348:g474

4. Sekula RF Jr, Frederickson AM, Jannetta PJ, Quigley MR, Aziz KM, Arnone GD. Microvascular decompression for elderly patients with trigeminal neuralgia: a prospective study and systematic review with meta-analysis. $J$ Neurosurg 2011;114:172-179

5. Barker FG $2^{\text {nd }}$, Jannetta PJ, Bissonette DJ, Larkins MV, Jho HD. The long-term outcome of microvascular decompression for trigeminal neuralgia. N Engl J Med 1996;334:1077-1083

6. Ugwuanyi UC, Kitchen ND. The operative findings in re-do microvascular decompression for recurrent trigeminal neuralgia. Br J Neurosurg 2010;24:26-30

7. Lee SH, Levy EI, Scarrow AM. Recurrent trigeminal neuralgia attributable to veins after microvascular decompression. Neurosurgery 2000;46:356-361

8. Gu W, Zhao W. Microvascular decompression for recurrent trigeminal neuralgia. J Clin Neurosci 2014;21:1549-1553

9. Amador N, Pollock BE. Repeat posterior fossa exploration for patients with persistent or recurrent idiopathic trigeminal neuralgia. $J$ Neurosurg 2008;108:916-920

10. Cho DY, Chang CG, Wang YC, Wang FH, Shen CC, Yang DY. Repeat Operations in Failed Microvascular Decompression for Trigeminal Neuralgia. Neurosurgery 1994;35:665-670 
11. Rath SA, Klein HJ, Richter, HP. Findings and Long-term Results of Subsequent Operations after Failed Microvascular Decompression for Trigeminal Neuralgia. Neurosurgery 1996;39:933-940

12. Liao JJ, Cheng WC, Chang $\mathrm{CN}$ et al. Reoperation for recurrent trigeminal neuralgia after microvascular decompression. Surg Neurol 1997;47:562-568

13. Huang CF, Chiou SY, Wu MF, Tu HT, Liu WS. Gamma Knife surgery for recurrent or residual trigeminal neuralgia after a failed initial procedure. J Neurosurg 2010;113 Suppl:172-7

14. Spatz AL, Zakrzewska JM, Kay EJ. Decision analysis of medical and surgical treatments for trigeminal neuralgia: how patient evaluations of benefits and risks affect the utility of treatment decisions. Pain 2007;131:302-310

15. Zakrzewska JM, Lopez BC, Kim SE, Coakham HB. Patient reports of satisfaction after microvascular decompression and partial sensory rhizotomy for trigeminal neuralgia. Neurosurgery 2005;56:13041311

16. Jafree DJ, Williams AC, Zakrzewska JM. Impact of pain and postoperative complications on patientreported outcome measures 5 years after microvascular decompression or partial sensory rhizotomy for trigeminal neuralgia. Acta Neurochir (Wien) 2018; 160:125-134.

17. Zakrzewska JM, Lopez BC, Kim SE, Varian EA, Coakham HB. Patient satisfaction after surgery for trigeminal neuralgia--development of a questionnaire. Acta Neurochir 2005;147:925-932

18. Headache Classification Committee of the International Headache Society. Classification and diagnostic criteria for headache disorders, cranial neuralgias and facial pain. Cephalgia 1998;8:1-96

19. Headache Classification Committee of the International Headache Society. The International Classification of Headache Disorders, 3rd edition. Cephalgia 2013;33:629-808

20. Cruccu G, Finnerup NB, Jensen TS et al. Trigeminal neuralgia: New classification and diagnostic grading for practice and research. Neurology 2016;87:220-228

21. Sindou M, Leston J, Decullier E, Chapuis F. Microvascular decompression for primary trigeminal neuralgia: long-term effectiveness and prognostic factors in a series of 362 consecutive patients with 
clear-cut neurovascular conflicts who underwent pure decompression. J Neurosurg 2007;107:11441153

22. Miller JP, Acar F, Burchiel KJ. Classification of trigeminal neuralgia: clinical, therapeutic, and prognostic implications in a series of 144 patients undergoing microvascular decompression. $J$ Neurosurg 2009;111:1231-1234

23. Theodosopoulos PV, Marco E, Applebury C, Lamborn KR, Wilson CB. Predictive model for pain recurrence after posterior fossa surgery for trigeminal neuralgia. Arch Neurol 2002;59:1297-1302

24. Tyler-Kabara EC, Kassam AB, Horowitz MH et al. Predictors of outcome in surgically managed patients with typical and atypical trigeminal neuralgia: comparison of results following microvascular decompression. J Neurosurg 2002;96:527-531

25. Jo KW, Kong DS, Hong KS, Lee JA, Park K. Long-term prognostic factors for microvascular decompression for trigeminal neuralgia. J Clin Neurosci 2013;20:440-445

26. Li ST, Pan Q, Liu N, Shen F, Liu Z, Guan Y. Trigeminal neuralgia: what are the important factors for good operative outcomes with microvascular decompression. Surg Neurol 2004;62:400-404

27. Leal PR, Barbier C, Hermier M, Souza MA, Cristino-Filho G, Sindou M. Atrophic changes in the trigeminal nerves of patients with trigeminal neuralgia due to neurovascular compression and their association with the severity of compression and clinical outcomes. $J$ Neurosurg 2014;120:1484-1495

28. Cheng J, Meng J, Liu W, Hui X, Lei D. Nerve atrophy in trigeminal neuralgia due to neurovascular compression and its association with surgical outcomes after microvascular decompression. Acta Neurochir (Wien) 2017;159:1699-1705.

29. Bakker NA, Van Dijk JM, Immenga S, Wagemakers M, Metzemaekers JD. Repeat microvascular decompression for recurrent idiopathic trigeminal neuralgia. $J$ Neurosurg 2014;121:936-939

30. Theodros D, Rory GC, Bender MT et al. Efficacy of primary microvascular decompression versus subsequent microvascular decompression for trigeminal neuralgia. J Neurosurg 2017;126:1691-1697

31. Burchiel KJ. A new classification for facial pain. Neurosurgery 2003;53:1164-1166 
32. Lee A, McCartney S, Burbidge C, Raslan AM, Burchiel KJ. Trigeminal neuralgia occurs and recurs in the absence of neurovascular compression. J Neurosurg 2014;120:1048-1054

33. Degn J, Brennum J. Surgical treatment of trigeminal neuralgia. Results from the use of glycerol injection, microvascular decompression, and rhizotomia. Acta Neurochir 2010;152:2125-2132

34. Sindou M, Leston J, Howeidy E, Decullier E, Chapuis F. Micro-vascular decompression for primary Trigeminal Neuralgia (typical or atypical). Long-term effectiveness on pain; prospective study with survival analysis in a consecutive series of 362 patients. Acta Neurochir 2006;148:1235-1245

35. Antonini G, Di PA, Cruccu G et al. Magnetic resonance imaging contribution for diagnosing symptomatic neurovascular contact in classical trigeminal neuralgia: a blinded case-control study and meta-analysis. Pain 2014;155:1464-14713

36. Ishikawa M, Nishi S, Aoki $\mathrm{T}$ et al. Operative findings in cases of trigeminal neuralgia without vascular compression: proposal of a different mechanism. J Clin Neurosci 2002:9;200-204

37. Revuelta-Gutierrez R, Lopez-Gonzalez MA, Soto-Hernandez JL. Surgical treatment of trigeminal neuralgia without vascular compression: 20 years of experience. Surg Neurol 2006;66:32-36

38. Gao J, Fu Y, Guo SK, Li B, Xu ZX. Efficacy and Prognostic Value of Partial Sensory Rhizotomy and Microvascular Decompression for Primary Trigeminal Neuralgia: A Comparative Study. Med Sci Monit 2017 23:2284-2291

39. Leung YY, McGrath C, Cheung LK. Trigeminal neurosensory deficit and patient reported outcome measures: the effect on quality of life. PLoS One 2013;8:e77391 


\section{APPENDIX}

Supplementary Table 1 | Vascular findings at MVD for patients with and without recurrence

\begin{tabular}{|c|c|c|}
\hline Operative finding & $\begin{array}{l}\text { Recurrence group } \\
\qquad(\mathrm{n}=26)\end{array}$ & $\begin{array}{l}\text { Recurrence free group } \\
\qquad(\mathrm{n}=141)\end{array}$ \\
\hline SCA $(\%)$ & $12(52.2)$ & $78(64.5)$ \\
\hline $\operatorname{AICA}(\%)$ & $5(21.7)$ & $29(24.0)$ \\
\hline Basilar artery (\%) & $1(4.3)$ & $0(0.0)$ \\
\hline Petrosal artery (\%) & $1(4.3)$ & $2(1.7)$ \\
\hline Unknown artery (\%) & $0(0.0)$ & $1(0.8)$ \\
\hline Mesencephalic vein (\%) & $1(4.3)$ & $3(2.5)$ \\
\hline Trigeminal vein $(\%)$ & $1(4.3)$ & $3(2.5)$ \\
\hline Unknown vein $(\%)$ & $0(0.0)$ & $3(2.5)$ \\
\hline Multiple veins (\%) & $1(4.3)$ & $2(1.7)$ \\
\hline
\end{tabular}

AICA, anterior inferior cerebellar arterior; SCA, superior cerebellar artery 


\section{TABLES}

Table 1 | Characteristics of patients with and without recurrence five years after MVD

\begin{tabular}{|c|c|c|c|}
\hline Characteristic & $\begin{array}{c}\text { Recurrence group } \\
(\mathrm{n}=\mathbf{2 8})\end{array}$ & $\begin{array}{l}\text { Recurrence free group } \\
\qquad(\mathrm{n}=141)\end{array}$ & Test; $p$ value \\
\hline $\begin{array}{l}\text { Median age in years } \\
\text { (IQR) }\end{array}$ & $59.0(46.3-70.8)$ & $60.0(51.0-69.0)$ & $U=1843.0 ; \mathrm{ns}$ \\
\hline Gender: female (\%) & $18(64.3)$ & $80(56.7)$ & $\chi^{2}=0.5 ; \mathrm{ns}$ \\
\hline $\begin{array}{l}\text { Median age at onset } \\
\text { (IQR) }\end{array}$ & $49.5(41.3-65.8)$ & $54.0(45.0-62.0)$ & $U=1158.0 ; \mathrm{ns}$ \\
\hline $\begin{array}{l}\text { Median duration of } \\
\text { symptoms in years (IQR) }\end{array}$ & $5.0(2.0-7.0)$ & $5.0(3.0-8.0)$ & $U=1168.5 ; \mathrm{ns}$ \\
\hline Division (\%) & & & $\chi^{2}=7.9 ; \mathrm{ns}$ \\
\hline$V 1$ & $1(3.6)$ & $7 / 124(5.6)$ & \\
\hline$V 2$ & $2(7.1)$ & $17 / 124(13.7)$ & \\
\hline$V 3$ & $6(21.4)$ & $26 / 124(21.0)$ & \\
\hline$V 1+V 2$ & $2(7.1)$ & $23 / 124(18.5)$ & \\
\hline$V 2+V 3$ & $1(3.6)$ & $12 / 124(9.7)$ & \\
\hline$V 1+V 2+V 3$ & $16(57.1)$ & $39 / 124(31.5)$ & \\
\hline $\begin{array}{l}\text { Side of pain (\%): } \\
\text { Right / Left }\end{array}$ & $18(64.3) / 10(35.7)$ & $\begin{array}{l}92 / 135(68.1) / 43 / 135 \\
(31.8)\end{array}$ & $\chi^{2}=0.2 ; \mathrm{ns}$ \\
\hline Operative findings $(\%)$ & & & $\chi^{2}=0.3 ; \mathrm{ns}$ \\
\hline Arterial compression & $17 / 25(68.0)$ & $103 / 129(64.8)$ & \\
\hline Venous compression & $4 / 25(16.0)$ & $23(14.5)$ & \\
\hline Mixed compression & $4 / 25(16.0)$ & $33(20.8)$ & \\
\hline
\end{tabular}




\section{FIGURE LEGENDS}

Fig. 1 | Patient flow diagram.

Fig. 2 Pain relief at five years after first MVD of medical, surgical and no treatment after recurrence of TN. MVD, microvascular decompression; PSR, partial sensory rhizotomy; RFT, radiofrequency thermocoagulation.

Supplementary Fig. 1 | Pain outcomes of those with failure of MVD. MVD, microvascular decompression; PSR, partial sensory rhizotomy; RFT, radiofrequency thermocoagulation; GI, glycerol injection.

Supplementary Fig. 2 | Pain outcomes of those with post-MVD recurrences after previous procedures. GI, glycerol injection. 
220 responded to the postal survey at fiveyear follow up

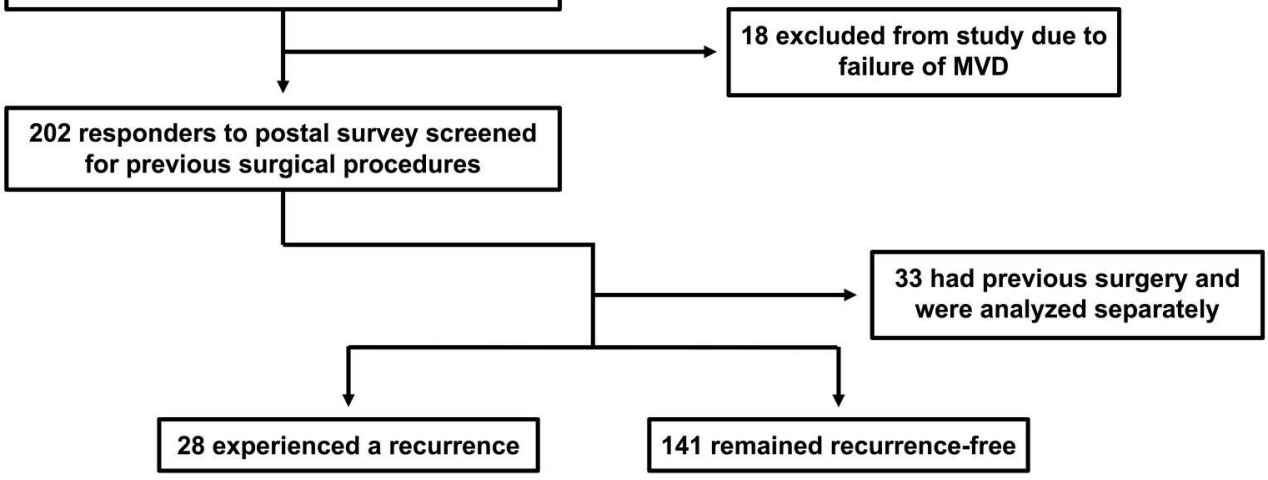

Fig. 1 | Patient flow diagram

$189 \times 110 \mathrm{~mm}(300 \times 300$ DPI $)$ 


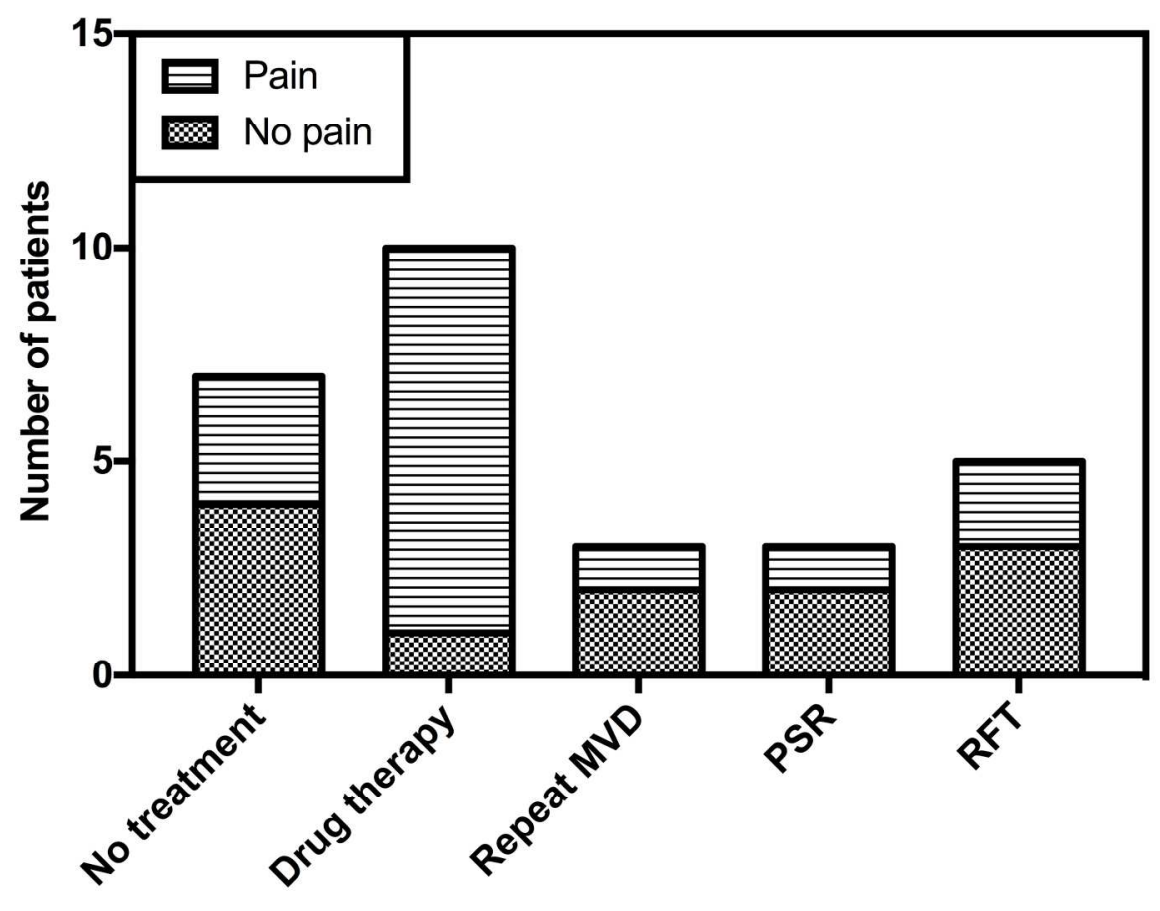

Fig. 2 | Pain relief at five years after first MVD of medical, surgical and no treatment after recurrence of TN. MVD, microvascular decompression; PSR, partial sensory rhizotomy; RFT, radiofrequency thermocoagulation $185 \times 134 \mathrm{~mm}(300 \times 300$ DPI) 


\section{8 patients experienced a} failure of their MVD

\begin{tabular}{|c|}
\hline 8/15 pain-free after \\
surgery \\
2/3 pain-free after MVD \\
4/4 pain-free after PSR \\
2/6 pain-free after RFT \\
0/2 pain-free after GI \\
\hline
\end{tabular}

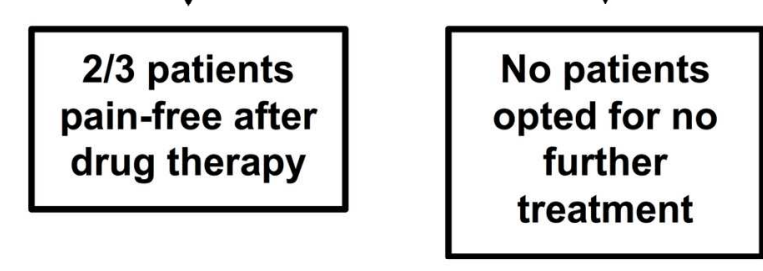



recurrence of their TN

4 peripheral surgery

7 Gasserion ganglion surgery

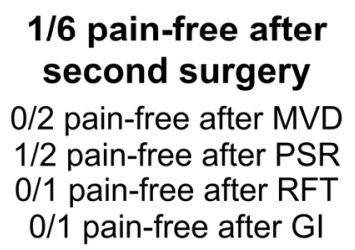

1/2 pain-free after no further therapy 